\title{
Shock wave lithotripsy in patients requiring anticoagulation or antiplatelet agents
}

\author{
Bader Alsaikhan, MD; Sero Andonian, MD, FRCSC
}

\begin{abstract}
Extracorporeal shockwave lithotripsy (SWL) remains the only truly minimally invasive procedure for the treatment of upper tract nephrolithiasis. Recently, there has been a recent rise in the patients on antiplatelet agents. Therefore, the aim of the present study is to review the literature available regarding SWL in these high-risk patients requiring anti-coagulation therapy. Alternative therapies to SWL are also presented.
\end{abstract}

Cite as: Can Urol Assoc J 2011;5(1)53-7; D01:10.5489/cuaj.09140

\section{Résumé}

La lithotripsie extracorporelle par ondes de choc (LECOC) demeure la seule intervention qui soit réellement minimalement invasive pour le traitement de la néphrolithiase dans les voies urinaires supérieures. Récemment, on a noté une hausse du nombre de patients traités par antiplaquettaires. Par conséquent, le but de la présente étude est d'examiner les articles publiés sur la LECOC chez ces patients à risque élevé ayant besoin d'un traitement anticoagulant. On présente également des traitements de rechange à la LECOC.

\section{Introduction}

Since its first clinical studies in West Germany in 1980s, the role of shock wave lithotripsy (SWL) in the treatment of renal stones has changed. ${ }^{1}$ The original Dornier Human Model-3 (HM-3) (Dornier Med Tech, Wessling, Germany) has largely been replaced with dry-head lithotripters; most of these are mobile, small and have an ultrasound unit in addition to a high quality fluoroscopy unit. In the latest 2007 American Urological Association Guidelines, SWL ceased to be the first-line therapy for upper- and mid-ureteral stones. ${ }^{2}$ Instead, ureteroscopy with holmium laser lithotripsy is the first-line therapy for these stones. This is due to technological advancement in miniaturization of ureteroscopes and the safety and efficacy of holmium laser energy in the treatment of various stone types. Nonetheless, SWL remains the first mode of therapy for small $(<2 \mathrm{~cm})$ renal stones and has an average stone-free rate of $82 \%$.

Since SWL is a minimally invasive procedure requiring intravenous sedation, many urologists and patients consider it as the preferred management option for small renal stones. ${ }^{3}$ Furthermore, its safety profile with low risk of side effects and its availability in most hospitals have contributed to its popularity. ${ }^{4}$ Classically, there have been two absolute contraindications for SWL: pregnancy and bleeding diathesis. Therefore, patients on either antiplatelet agents or full anticoagulation therapy with warfarin are usually managed with other modes of therapy or their anticoagulation agents are held perioperatively. This is because the use of antithrombotic agents possesses a high risk of hemorrhagic complications, such as serious postoperative peri-renal hematomas and renal hemorrhage, leading to protracted postoperative course and possibly requiring drastic measures, such as nephrectomy or renal embolization. ${ }^{5-8}$ The aim of this article is to present data regarding SWL in anticoagulated patients and to present alternatives to SWL.

\section{Methods}

A MEDLINE search was performed for original articles from August 1987 to February 2010. MeSH headings of "lithotripsy" in combination with "anticoagulants," "aspirin," "platelet aggregation inhibitors," "hematoma" or "drug-eluting stents" were used. Due to the paucity of retrieved articles, key references were also included. To further expand the search parameters, original articles describing other modes of lithothripsy (ureteroscopy and percutaneous nephrolithotomy) in anticoagulated patients were included. A total of 58 articles were reviewed. Sixteen articles were discarded due to irrelevance, the presence of more recent studies, or the presence of articles with stronger levels of evidence.

\section{Results}

Prospective studies, systematic reviews and case reports were reviewed. The quality and strength of the evidence in the identified articles were generally poor, due to the lack of Level I evidence. There have been no randomized controlled trials evaluating SWL in patients requiring anticoagulation therapy. Safety and duration of anticoagulation discontinuation have been based on expert opinion rather than scientific evidence. 
The most common side effect of SWL is hematuria; the treatment results in microtrauma to the renal tissue. ${ }^{9-11}$ With the HM3 electrohydraulic lithotripter, the incidence of post-SWL symptomatic peri-renal hematomas detected by ultrasound is estimated to be $0.1 \%$ to $0.6 \% .{ }^{12-14}$ With the use of more sensitive abdominal computed tomography scanning, the rate of asymptomatic peri-renal hematomas is up to $25 \% .{ }^{15,16}$ Although a retrospective series of $5(0.49 \%)$ patients with symptomatic peri-renal hematomas found that all 5 patients were hypertensive, 3 had diabetes mellitus, and 2 had coronary artery disease, these risk factors have not been consistently demonstrated with the exception of uncontrolled hypertension. ${ }^{13,17}$ Recently, an ultrasound examination of all patients post-SWL with the electromagnetic Storz Modulith SLX lithotripter (Storz, St. Louis, MO) found the incidence of asymptomatic peri-renal hematomas to be $4.1 \%$, while the incidence of symptomatic peri-renal hematomas was similar to other lithotripters at a rate of $0.7 \% .^{18}$ Furthermore, the probability of hematoma increased 1.67 times for every 10 -year increase in patient age.$^{18} \mathrm{In}$ terms of lithotripter used, the electrohydraulic HM3 was found to cause more trauma than electromagnetic, which in turn was found to cause more trauma than the piezoelectric lithotripter. ${ }^{19-21}$ Furthermore, in animal studies, slow frequency SWL appears to be associated with reduced renal injury and hematoma formation. ${ }^{22}$ Therefore, increasing patient age, uncontrolled hypertension, type of lithotripter (electrohydraulic $>$ electromagnetic $>$ piezoelectric) and fast rate have been associated with renal trauma and peri-renal hematomas.

\section{Patients without drug-eluting stents requiring antiplatelet agents}

Zanetti and colleagues prospectively evaluated the safety of discontinuation of antithrombotic therapy with or without the institution of bridging anticoagulation therapy in patients undergoing SWL with Storz Modulith SLX lithotripter (Storz, St. Louis, MO). ${ }^{7}$ This is Level III evidence according to the Cochrane classification. Twenty-three patients taking antiplatelet agents (acetylsalicylic acid [ASA], ticlopidine, dipyridamole) were stratified according to author-defined risk levels. Patients considered to be at "low-risk of thrombotic events" (previous myocardial infarction more than 1 year ago) had discontinued antiplatelet therapy 8 days prior to SWL and a resumption of therapy 10 to 14 days post-SWL. Patients considered to be at "high-risk of thrombotic events" (coronary artery bypass graft [CABG], atrial fibrillation, cerebrovascular disease and peripheral occlusive arterial disease) discontinued antiplatelet therapy 8 days prior to SWL with substitution with unfractionated heparin 5000 units 3 times a day. This was continued postoperatively for up to 10 to 14 days. Both groups were followed with abdominal ultrasound and serial hemoglobin measurements. In both groups, there were no hemorrhagic complications. ${ }^{7}$ The advantages of this approach is that multiple sessions of SWL could be performed while holding heparin dose on the morning of the procedure. However, there were no patients in this series requiring full anticoagulation therapy or antiplatelet agents, such as those with drug-eluting coronary stents. Therefore, none of these patients would be considered high-risk as defined later.

\section{Patients with drug-eluting stents requiring antiplatelet agents}

Drug-eluting stents are inserted during percutaneous coronary angiography, and the presence of these stents necessitate prolonged use of clopidogrel as the early discontinuation of the medication leads to increased risk of stent thrombosis. ${ }^{23}$ Normally, coronary artery stents undergo re-endothelization. This re-endothelization period is 4 to 6 weeks in bare-metal stents (BMSs), while in drug eluting stents it may take up to 1 year. ${ }^{24}$ The maximal risk of thrombosis occurs during this period of re-endothelization, and the risk of stent thrombosis increases when antiplatelet agents are suddenly withheld. ${ }^{25}$ Stent thrombosis has been reported when ASA is discontinued even beyond 1 year after drug-eluting stent insertion. ${ }^{26}$ However, clopidogrel is the main antiplatelet agent associated with drug-eluting stent thrombosis when withdrawn. ${ }^{27}$ Therefore, elective surgical procedures should be ideally delayed for 6 months to 1 year after insertion of these stents. ${ }^{24}$

There are serious clinical consequences to discontinuing anticoagulation therapy post-placement of coronary artery stents and these are worse after the modern drug-eluting stents. Several studies reported stent thrombosis after premature discontinuation of antiplatlet therapy. Iakovou and colleagues reported 29\% incidence of drug-eluting stent thrombosis after premature discontinuation of antiplatelet therapy. ${ }^{25}$ At 11 months, the mortalitiy rate was $7.5 \%$ in patients who discontinued theinopyridine therapy versus only $0.7 \%$ in patients continuing the treatment. ${ }^{28}$ For patients with BMSs undergoing non-cardiac surgical procedures, 7 out of 40 (18\%) developed myocardial infarction (MI); 5 out of the 7 had discontinued thienopyridine therapy and 6 out of the 7 Mls were fatal. ${ }^{29}$ Therefore, discussion with the interventional cardiologist is important to weigh the risks and benefits of discontinuation of antiplatelet agents versus delaying elective surgical procedures.

A science advisory (comprised of the American Heart Association [AHA], American College of Cardiology [ACC], Society for Cardiovascular Angiography and Interventions [SCAI], American College of Surgeons [ACS] and American Dental Association [ADA]) emphasized the importance of 12 months of dual antiplatelet therapy after the insertion of drug-eluting stents and recommended postponement of elective surgical procedures until the completion of the appropriate course of thienopyridine therapy. ${ }^{30}$ For those cases where 
the surgical procedure cannot be postponed, the advisory committee recommended the continuation of ASA perioperatively in high-risk patients, and to restore thienopyridine treatment as soon as possible postoperatively. ${ }^{30}$ The AHA/ ACC/SCAI recommended that, at minimum, patients with bare-metal stents should be treated with clopidogrel $75 \mathrm{mg}$ and ASA $325 \mathrm{mg}$ for 1 month after insertion, 3 months after sirolimus eluting stent implantation, 6 months after paclitaxel eluting stent implantation, and up to 12 months in patients who are not at a high risk of bleeding. ${ }^{30}$

There are no clear guidelines for patients requiring antiplatelet agents and SWL. Severe life-threatening complications have been reported in patients taking clopidogrel and undergoing SWL. ${ }^{31}$ Katz and colleagues reported on a patient who developed life-threatening retroperitoneal hematoma when he required intravenous heparin therapy for post-SWL MI. ${ }^{32}$

Di Minno and colleagues proposed a guideline to manage patients on antiplatelet agents undergoing surgical procedures. ${ }^{24} \mathrm{~A}$ management guideline is proposed according to the thrombotic risk of the patient and the hemorrhagic risk of the surgical procedure (Table 1). In this guideline, surgical procedures have been classified into low hemorrhagic risk (superficial procedures), intermediate hemorrhagic risk (such as transurethral procedures) and high hemorrhagic risk (with possible bleeding in a closed space such as intracranial neurosurgical procedures). Furthermore, patients are classified into low, intermediate and high cardiovascular disease (CVD) risk according to the timing of cardiovascular events and types of coronary stents used.

In high CVD risk patients (i.e. 6 weeks after MI, percutaneous coronary intervention [PCI], BMSs, CABG; 6 months after same if complicated; 12 months after highrisk drug-eluting stent; 2 weeks after stroke), it is recommended to postpone all elective surgical procedures and perform only emergency procedures while maintaining on ASA and clopidogrel (for low and intermediate hemorrhagic risk procedures) or ASA combined with triofiban/eptifibatide and heparin bridging (for high hemorrhagic risk procedures). In intermediate CVD risk patients (i.e. 12 months after the drug-eluting stent; low ejection fraction, diabetes mellitus, high-risk stents), it is recommended to conduct elective low hemorrhagic risk procedures while on ASA and clopidogrel (if prescribed), and postpone elective intermediate and high hemorrhagic risk procedures. If these procedures can not be postponed, it is recommended to maintain ASA and clopidogrel for intermediate hemorrhagic risk procedures while keeping patients on ASA only for high hemorrhagic risk procedures. Clopidogrel should be re-started 12 to 24 hours postoperatively. In low CVD risk patients (i.e., 6 months after MI, PCl, BMS, CABG and stroke; 12 months if with complications), it is advisable to continue ASA except in high hemorrhagic risk procedures, such as SWL, when ASA is withdrawn and statin is continued. ${ }^{24}$
These recommendations were not specific to a certain surgical procedure, and SWL was not mentioned in the Di Minno study. Since the kidney is enclosed within Gerota's fascia and peri-renal hematomas can cause mass effect, SWL is considered a high hemorrhagic risk procedure. Therefore, in low CVD risk patients, SWL could be performed while continuing statins and withholding ASA (maximum 7 days) after consultation with cardiology, while in intermediate and high CVD risk patients, SWL should be postponed or other alternatives be used. Prophylactic therapy with subcutaneous unfractionated heparin as a bridge to the discontinuation of antiplatelet agents has been successful in one study. ${ }^{7}$ However, antiplatelet treatment replacement by heparin or low-molecular weight heparin does not provide protection against the risk of coronary artery or stent thrombosis since heparin does not have antiplatelet properties, as it inactivates thrombin and activated Factor $X$ (Factor Xa) via an antithrombin dependent mechanism, whereas clopidogrel is an ADP-receptor antagonist and ASA inhibits production of thromboxane by inhibiting cyclooxygenase. ${ }^{24,33}$

\section{Alternatives to SWL in anticoagulated patients}

Similar to SWL, percutaneous nephrolithotomy (PCNL) is contraindicated in patients with uncorrected coagulopathy. ${ }^{34}$ However, there is a recent retrospective series from the Cleveland Clinic Foundation of 27 high-risk patients on anticoagulation therapy (warfarin, clopidogrel or cilostazol) who underwent percutaneous nephrolithotomy. ${ }^{35}$ Warfarin was withheld 5 days preoperatively with enoxaparin (low molecular weight heparin) bridging and resumed 5 days postoperatively. Clopidogrel and cilostazol were stopped 10 days preoperatively and resumed 5 days postoperatively. In that series, the stone-free rate was $93 \%$. There were 2 patients (7\%) with significant bleeding and 1 patient $(4 \%)$ developed a thromboembolic complication. Therefore, PCNL could be performed in these high-risk patients after careful consultation with the prescribing cardiologist and with perioperative bridging with enoxaprin with acceptable rates of postoperative complications.

The safest alternative to SWL in these high risk patients, however, is ureteroscopy and holmium:YAG laser lithotripsy. There are 3 retrospective series confirming the safety of ureteroscopy and use of holmium laser energy in patients with uncorrected bleeding diatheses. In the first series, 9 patients (with mean stone size of $9.5 \mathrm{~mm}$, range $4-15 \mathrm{~mm}$ ) were treated successfully with 1 patient developing postoperative oliguria due to small ureteral clot that resolved spontaneously. Another patient developed epistaxis after administration of ketorolac for pain. ${ }^{36}$ The second larger study is from the University of Western Ontario. This retrospective study included 25 patients with 29 upper tract calculi who underwent ureteroscopic holmium laser lithotripsy. The 
Table 1. Guidelines in management of patients on anti-platelet agents requiring elective surgery according to risk level

\section{Patient risk level}

I) Low CVD risk:

- 6 months after $\mathrm{MI}, \mathrm{PCl}, \mathrm{BMS}, \mathrm{CABG}$, stroke

- 12 months if with complications

II) Intermediate CVD risk:

- 12 months after DES

- low ejection fraction

- diabetes mellitus

- high-risk stents (long, proximal, multiple,

overlapping, small vessels, bifurcation

\section{Surgical hemorrhagic risk}

A) Low (superficial such as circumcision)

B) Intermediate (such as transurethral cases)

C) High (possible bleeding in a closed space such as SWL)

A) Low (superficial such as circumcision)

\section{Suggestion}

Proceed with elective surgery on ASA
Proceed with elective surgery on ASA

Proceed with elective surgery on statin; Withdraw ASA (maximum 7 days)

Proceed with elective surgery on ASA Maintain clopidogrel (if prescribed)
B) Intermediate (such as transurethral cases)

C) High (possible bleeding in a closed space such as SWL)

A) Low (superficial such as circumcision)

III) High CVD risk:

- 6 weeks after $\mathrm{MI}, \mathrm{PCl}, \mathrm{BMS}, \mathrm{CABG}$

- 6 months after same if complications

- 12 months after high-risk DES

- 2 weeks after stroke
Postpone elective surgery If surgery absolutely required: Maintain ASA and clopidogrel (if prescribed)

Postpone elective surgery If surgery absolutely required: Maintain ASA and stop clopidogrel

Postpone elective surgery If surgery absolutely required: Maintain ASA and clopidogrel

B) Intermediate (such as transurethral cases)

C) High (possible bleeding in a closed space such as SWL)
Postpone elective surgery If surgery absolutely required: Maintain ASA and clopidogrel

Postpone elective surgery

If surgery absolutely required: maintain aspirin; bridge with tirofiban/eptifibatide and heparin

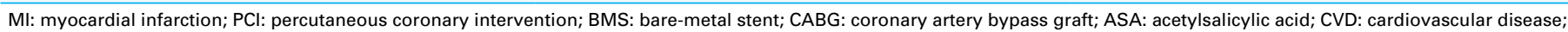
DES: drug-eluting stent; SWL: shock wave lithotripsy.

Adapted from Di Minno et al., 2009. ${ }^{24}$

mean stone size was $11.9 \mathrm{~mm}$ (range 6-25 mm). Bleeding diatheses involved warfarin administration for various conditions in 17 patients, liver dysfunction in 3, thrombocytopenia in 4 and von Willebrand's disease in 1 . Stone-free rate was $96 \%$ (27 of the 28 ) and 29 of 30 procedures were completed successfully without significant complication. In a patient treated concomitantly with electrohydraulic lithotripsy, significant retroperitoneal hemorrhage developed and the patient required a blood transfusion. ${ }^{37}$ In a case-control study comparing 37 anticoagulated patients to controls undergoing ureteroscopy and laser lithotripsy, there were no increased risk of hemorrhagic complications. ${ }^{38}$ In this study, the mean stone size was $13.2 \mathrm{~mm}$ (range 5-35 mm). Therefore, ureteroscopy and holmium laser lithotripsy are the safest modalities in these intermediate- and high-risk patients requiring perioperative antiplatelet agents when these elective procedures could not be postponed.

The present review article is meant to be descriptive. There was lack of data to be analyzed in a systematic fashion. Therefore, there may have been studies not included in the present review.

\section{Conclusion}

Anticoagulation remains a contraindication for SWL. In lowrisk patients requiring $S W L$, it is reasonable to withhold their antiplatelet agents perioperatively (maximum 7 days) after consultation with the prescribing cardiologist and informing patients of risks of hemorrhage and thrombotic events. Substitution with unfractionated heparin, although reported in one study, is not recommended since heparin does not have the same properties as antiplatelet agents. Intermediateand high-risk patients should be managed individually with a high level of caution. Ideally, elective surgery should be postponed 6 to 12 months and only emergent procedures performed while maintaining patients on antiplatelet agents. There are no randomized controlled trials in this field as they are difficult to perform due to the requirement of many patients since the incidence of clinically significant peri-renal hematomas is low. Ureteroscopy and laser lithotripsy while patients are on anticoagulation therapy remain the safest modalities when these procedures could not be postponed. 
Department of Urology, McGill University Health Centre, Montreal, QC

Acknowledgements: This work was supported in part by the Northeastern AUA Young Investigator Award and McGill University Health Centre Foundation Award to Sero Andonian.

Competing interests: None declared.

This paper has been peer-reviewed.

\section{References}

1. Chaussy C, Schmiedt E, Jocham D, et al. First clinical experience with extracorporeally induced destruction of kidney stones by shock waves, 1981. J Urol 2002;167:1957-60.

2. Preminger $\mathrm{GM}$, Tiselius $\mathrm{HG}$, Assimos DG, et al. 2007 guideline for the management of uretral calculi. J Urol 2007; 178:2418-34.

3. Streem SB, Yost A. Extracorporeal shock wave lithotripsy in patients with bleeding diatheses. I Urol 1990;144:1347-8.

4. Sighinolfi MC, Micali S, Grande M, et al. Extracorporeal shock wave lithotripsy in an elderly population: how to prevent complications and make the treatment safe and effective. J Endourol 2008;22:2223-6.

5. Ruiz H, Saltzman B. Aspirin-induced bilateral renal hemorrhage after extracorporeal shock wave lithotripsy: implications and conclusions. J Urol 1990;143:791-2.

6. Knorr PA, Woodside JR. Large perirenal hematoma after extracorporeal shock-wave lithotripsy. Urology 1990;35:151-3.

7. Zanetti G, Kartalas-Goumas I, Montanari E, et al. Extracorporeal shockwave lithotripsy in patients treated with antithrombotic agents. J Endourol 2001;15:237-41.

8. Sare GM, Lloyd FR, Stower MJ. Life-threatening haemorrhage after extracorporeal shockwave lithotripsy in a patient taking clopidogrel. BJU Int 2002;90:469.

9. Baumgartner BR, Dickey KW, Ambrose SS, et al. Kidney changes after extracorporeal shock wave lithotripsy: Appearance on MR imaging. Radiology 1987;163:531-4.

10. Lingeman JE, Smith LH, Woods JR, Newman DM (editors). Urinary calculi: ESWL, Endourology and Medical Therapy. Philadelphia, PA: Lea \& Febiger; 1989.

11. Eisenberger F, Miller K, Rassweiler J (editors). Stone Therapy in Urology. Stuttgart: Thieme; 1991.

12. Chaussy C, Schuller J, Schmiedt E, et al. Extracorporeal shock-wave lithotripsy (ESWL) for treatment of urolithiasis. Urology 1984;23:59-66.

13. Knapp PM, Kulb TB, Lingeman JE, et al. Extracorporeal shock wave lithotripsy-induced perirenal hematomas. J Urol 1988;139:700-3.

14. Cass AS, Brooker W, Duthoy E, et al. Clinically diagnosed renal hemorrhage after extracorporeal shock wave lithotripsy with Dornier HM3 and Medstone lithotripters. J Endourol 1992;6:413.

15. Kaude JV, Williams CM, Millner MR, et al. Renal morphology and function immediately after extracorporeal shock-wave lithotripsy. AJR Am J Roentgenol 1985; 145:305-13.

16. Rubin Jl, Arger PH, Pollack HM, et al. Kidney changes after extracorporeal shock wave lithotripsy: CT evaluation. Radiology 1987;162:21-4.

17. Newman LH, Saltzman B. Identifying risk factors in development of clinically significant post-shock-wave lithotripsy subcapsular hematomas. Urology 1991;38:35-8.

18. Dhar NB, Thornton J, Karafa MT, et al. A multivariate analysis of risk factors associated with subcapsular hematoma formation following electromagnetic shock wave lithotripsy. J Urol 2004;172:2271-4.
19. Skolarikos A, Alivizatos $G$, de la Rosette J. Extracorporeal shock wave lithotripsy 25 years later: complications and their prevention. Eur Urol 2006;50:981-90.

20. Momose A, Funyu T, Takahashi $\mathrm{N}$, et al. Effect of pressure distribution of shockwave on renal hemorrhage after extracorporeal shockwave lithotripsy: comparison of EDAP LT-01 and Siemens Lithostar. J Endourol 1999;13:165-71.

21. Graber SF, Danuser H, Hochreiter WW, et al. A prospective randomized trial comparing 2 lithotriptors for stone disintegration and induced renal trauma. J Urol 2003;169:54-7.

22. Evan AP, McAteer JA, Connors BA, et al. Renal injury during shock wave lithotripsy is significantly reduced by slowing the rate of shock wave delivery. BJU Int 2007;100:624-7.

23. Schulz S, Schuster T, Mehilli J, et al. Stent thrombosis after drug-eluting stent implantation: incidence, timing, and relation to discontinuation of clopidogrel therapy over a 4-year period. Eur Heart J 2009;30:2714-21.

24. Di Minno MN, Prisco D, Ruocco AL, et al. Perioperative handling of patients on antiplatelet therapy with need for surgery. Intern Emerg Med 2009;4:279-88.

25. Iakovou I, Schmidt T, Bonizzoni E, et al. Incidence, predictors, and outcome of thrombosis after successful implantation of drug-eluting stents. JAMA 2005;293:2126-30.

26. McFadden EP, Stabile E, Regar E, et al. Late thrombosis in drug-eluting coronary stents after discontinuation of antiplatelet therapy. Lancet 2004;364:1519-21.

27. Ong AT, McFadden EP, Regar E, et al. Late angiographic stent thrombosis (LAST) events with drug-eluting stents. J Am Coll Cardiol 2005;45:2088-92.

28. Spertus JA, Kettelkamp R, Vance C, et al. Prevalence, predictors, and outcomes of premature discontinuation of thienopyridine therapy after drug-eluting stent placement: results from the PREMIER registry. Circulation 2006;113:2803-9.

29. Kaluza GL, Joseph J, Lee JR, et al. Catastrophic outcomes of noncardiac surgery soon after coronary stenting. J Am Coll Cardiol 2000;35:1288-94.

30. Grines $\mathrm{CL}$, Bonow RO, Casey DE Jr, et al. Prevention of premature discontinuation of dual antiplatelet therapy in patients with coronary artery stents: a science advisory from the American Heart Association, American College of Cardiology, Society for Cardiovascular Angiography and Interventions, American College of Surgeons, and American Dental Association, with representation from the American College of Physicians. J Am Coll Cardiol 2007;49:734-9.

31. Sare GM, Lloyd FR, Stower MJ. Life-threatening haemorhage after extracorporeal shockwave lithotripsy in a patient taking clopidogrel. BJU Int 2002;90:469.

32. Katz R, Admon D, Pode D. Life-threatening retroperitoneal hematoma caused by anticoagulant therapy for myocardial infarction after SWL. J Endourol 1997;11:23-5.

33. Hirsh J, Anand SS, Halperin JL, et al. Mechanism of action and pharmacology of unfractionated heparin. Arterioscler Thromb Vasc Biol 2001;7:1094-6.

34. Andonian S, Okeke Z, Smith AD. Complications of Percutaneous Renal Surgery. AUA Update Series. Lesson 26. 2008.

35. Kefer JC, Turna B, Stein RJ, et al. Safety and efficacy of percutaneous nephrostolithotomy in patients on anticoagulant therapy. J Urol 2009;181:144-8.

36. Kuo RL, Aslan P, Fitzgerald KB, et al. Use of ureteroscopy and holmium:YAG laser in patients with bleeding diatheses. Urology 1998;52:609-13.

37. Watterson JD, Girvan AR, Cook AJ, et al. Safety and efficacy of holmium: YAG laser lithotripsy in patients with bleeding diatheses. J Urol 2002;168:442-5.

38. Turna B, Stein RJ, Smaldone MC, et al. Safety and efficacy of flexible ureterorenoscopy and holmium:YAG lithotripsy for intrarenal stones in anticoagulated cases. J Urol. 2008;179:1415-9.

Correspondence: Dr. Sero Andonian, Assistant Professor of Urology, Royal Victoria Hospital, McGill University Health Centre, 687 ave des Pins Ouest, Suite S6.92, Montreal, QC H3A 1A1; fax: 514843-1552; sero.andonian@muhc.mcgill.ca 